\title{
La preuve de Dieu par les Pygmées
}

Le laboratoire équatorial d'une ethnologie catholique

The Pygmies' Proof of God. The Equatorial Laboratory of Catholic Ethnology

André Mary

\section{OpenEdition}

\section{Journals}

Édition électronique

URL : https://journals.openedition.org/etudesafricaines/16411

DOI : 10.4000/etudesafricaines. 16411

ISSN : $1777-5353$

Éditeur

Éditions de l'EHESS

Édition imprimée

Date de publication : 20 novembre 2010

Pagination : 881-905

ISBN : 978-2-7132-2252-8

ISSN : 0008-0055

Référence électronique

André Mary, "La preuve de Dieu par les Pygmées », Cahiers d'études africaines [En ligne], 198-199-200 |

2010, mis en ligne le 02 janvier 2013, consulté le 21 avril 2022. URL : http://journals.openedition.org/ etudesafricaines/16411; DOI : https://doi.org/10.4000/etudesafricaines.16411

Ce document a été généré automatiquement le 21 avril 2022.

(c) Cahiers d'Études africaines 


\title{
La preuve de Dieu par les Pygmées
}

\author{
Le laboratoire équatorial d'une ethnologie catholique \\ The Pygmies' Proof of God. The Equatorial Laboratory of Catholic Ethnology
}

André Mary

1 Les études linguistiques et les observations ethnographiques des missionnaires, travaux d'amateurs ou inspirés par les méthodes et l'esprit scientifique de l'ethnologie universitaire, ont alimenté de longue date les travaux des anthropologues professionnels. Evans-Pritchard, dans son introduction à Sorcellerie, oracles et magie chez les Azande va jusqu'à remercier les missionnaires de la Church Missionary Society, non seulement de lui avoir préparé une "grammaire » zandé, mais aussi d'avoir éduqué les Azandé pour lui fournir des informateurs : "S'ils n'avaient pas consacré leurs vies à éduquer les Azandé, je n'aurais pas eu le bonheur d'être aidé par mon commis Zandé, qui a rédigé pour moi bien des textes originaux » (Evans-Pritchard 1972 : 32-33). Plus rare, il invite les lecteurs, au moment où il publie son livre, à lire en parallèle, l'excellente monographie de $\mathrm{M}^{\mathrm{gr}}$ Lagae sur Les Azandé ou Niam-Niam (1926) qui n'aura pas, il faut le dire, la même postérité. Dans Tristes Tropiques, C. Lévi-Strauss (1962 : 251) reconnaît également, dans un passage rarement cité, sa dette ethnographique envers les Salésiens, "nos meilleures sources", et particulièrement envers "l'indien du pape», son informateur et professeur de sociologie bororo, éduqué par les missionnaires et reçu à Rome, mais retourné entre-temps à la vie exemplaire du parfait sauvage à la suite d'une crise spirituelle.

2 Plus récemment, les anthropologues et les historiens ont pris la mesure de toutes les ressources ethnohistoriques mais aussi ethnographiques des journaux de bord tenus par les missionnaires européens ou indigènes, catéchistes ou évangélistes, répondant à la demande d'information de l'autorité institutionnelle de leur congrégation. La question des modes de lecture et de l'exploitation scientifique de ces récits missionnaires (missionnary narratives) a alimenté un vaste débat méthodologique entre historiens anthropologues et anthropologues historiens ${ }^{1}$. Mais le débat est aussi épistémologique puisque le travail ethnographique et l'investissement anthropologique de certains missionnaires, souvent isolés, obéissaient autant à une passion personnelle qu'aux incitations de leurs supérieurs à rechercher les pierres d'attente ou les jalons du 
message évangélique. On connaît la formule : «Comprendre pour être compris. » De la traduction des catéchismes ou de la Bible en langue vernaculaire, à la production de traités savants sur la parenté ou la religion primitive, en passant par l'élaboration érudite de dictionnaires qui sont parfois de véritables encyclopédies, ces missionnaires ethnologues ont apporté une contribution majeure à l'émergence de cette discipline naissante qu'était l'ethnologie. Certaines figures connues (Aupiais, Leenhardt) se sont mis à l'école des grands maîtres de cette discipline savante et universitaire, et notamment de Mauss. En retour, la production écrite des missionnaires de terrain entre en concurrence avec celle des administrateurs coloniaux dans la collaboration parfois conflictuelle avec les anthropologues professionnels. Le problème du statut épistémologique de cette ethnographie missionnaire se pose particulièrement dans le cadre de la formation des catégories de l'anthropologie religieuse et reste plus que jamais d'actualité.

3 Le questionnement qui est au cœur de cette contribution porte sur la production à la fois savante et militante d'une anthropologie apologétique parfaitement au fait de la culture universitaire et scientifique de son époque. Les appellations d'ethnologie ou de sociologie "religieuse", comme celle plus large de "sciences religieuses", entretiennent la confusion entre projet scientifique et préoccupations religieuses. P. Bourdieu (1987: 110) dénonçait, à sa façon, toutes ces entreprises qui prétendent cumuler les profits de la religiosité et de la scientificité au service d'une "science édifiante " ou d'une "religiosité savante ». On sait qu'après avoir perçu l'émergence des sciences des religions comme une entreprise "d'irréligion", beaucoup de professionnels des religions, et les institutions religieuses qui les employaient, ont fait le choix de s'approprier ces savoirs et même de les produire eux-mêmes soit pour accommoder la transmission du message évangélique soit pour s'engager dans une véritable contre-science. Ces entreprises de contre-culture trouvent un large écho et une complicité, non sans malentendu, dans la relecture post-moderniste et relativiste des écrits de la tradition anthropologique mais le problème de la fiabilité et de la crédibilité ethnographique de ces opérations apologétiques mérite d'être posé et abordé de front.

L'exemple singulier sur lequel je propose de revenir est l'élaboration, à l'instigation du fameux père Schmidt, le fondateur de la revue Anthropos - dont on a célébré le centenaire en 2005 -, d'une "ethnologie religieuse catholique ", prenant le contrepied des théories de l'ethnologie universitaire de Mauss et de l'histoire «laïque » des religions enseignée alors à l'École pratique des hautes études. Les travaux importants de $\mathrm{M}^{\mathrm{gr}}$ Le Roy et du R. P. Trilles sur la religion "primitive» des Pygmées d'Afrique équatoriale sont une des applications parmi d'autres de ce programme d'une ethnologie religieuse qui se voulait sans préjugé métaphysique, athée, matérialiste ou évolutionniste, engagé par des missionnaires, connaissant le terrain, les langues et les populations, et qui a fait des campements de "nos petits Négrilles» une sorte de "terrain apologétique» exceptionnel et unique. Cette grande entreprise savante se tient au départ à distance des préoccupations d'une missiologie qui était parallèlement en train de se constituer dans les années 1910 comme discipline pratique autonome (Vandenberghe 2006) mais, entre les années 1920 et 1930, la rencontre entre les intérêts missiologiques et les recherches ethnologiques s'est institutionnalisée, avec le soutien du Vatican, à travers l'Institut Anthropos. L'ironie est que les spécialistes de l'histoire de la missiologie ont souligné depuis à quel point l'entreprise de Schmidt avait finalement peu à voir avec le souci d'une anthropologie appliquée, à vocation 
missiologique, et ne pouvait pas de toute façon apporter une quelconque contribution aux problématiques de l'«adaptation » du message chrétien aux cultures indigènes, puisque les présupposés théologiques de la révélation primitive n'attendent absolument rien de l'apport de ces cultures (Dietrich 1992). Ce sont à l'inverse pour Schmidt les missionnaires qui sont censés fournir les informateurs de la nouvelle science, sans préjugé et donc sans connaissance des fausses théories ethnologiques, et donc sans contradiction possible.

\section{Le montage d'un terrain apologétique}

5 Dans mes travaux sur le bwiti des Fang du Gabon, et le travail syncrétique des prophètes de la religion d'Eboga, j'ai été conduit à m'intéresser à la situation d'interaction première de la rencontre missionnaire qui a précédé largement les mouvements prophétiques et les cultes syncrétiques indigènes de la période coloniale des années 1930 (Mary 1999). Le travail missionnaire de bricolage des héros des traditions fang qui conduit dans la seconde moitié du XIX à l'invention du dieu Nzame, en un mot à la promotion d'un dieu-ancêtre de la généalogie tribale ou d'un chef de village légendaire au statut d'Être suprême ou de Dieu "en personne ", a pu être qualifié de «manipulation missionnaire» (Mbot 1975: 112). L'expression n'est pas fausse, mais il est plus productif d'y voir a posteriori, pour reprendre le concept de Marshall Sahlins (1979), une sorte de « working misunderstanding », associant le travail de l'incompréhension de l'autre à un certain génie indigène du bricolage religieux.

6 Le démontage des erreurs commises par les missionnaires dans leur quête d'une appellation divine indigène adaptée à la figure du dieu dont ils étaient porteurs est un lieu commun des écrits ethnologiques. Les traductions en langue vernaculaire du message biblique et les premiers catéchismes missionnaires pratiquent de fait une manipulation des panthéons locaux et des étymologies qui suscitent depuis longtemps l'ironie mordante des ethnologues. L'exercice aboutit sans grande difficulté à dénoncer les contresens liés entre autres à l'adoption de noms d'emprunt isolés de leur contexte de pertinence ou à l'ignorance de l'écart des systèmes religieux en présence ${ }^{2}$. Mais la posture de la dénonciation des montages de l'entreprise missionnaire peut ellemême alimenter quelque malentendu. Dans la plupart des cas, la mésinterprétation s'explique difficilement par les œillères de l'action pastorale: les traducteurs ont souvent une vision éclairée des conceptions indigènes du monde, mobilisent les théories savantes de l'époque, et témoignent d'une fréquentation de longue durée des populations dont la majorité des anthropologues de terrain ne sauraient se prévaloir. La critique anthropologique laisse surtout entendre, au moins implicitement, que l'incompréhension missionnaire aurait pu être évitée : au-delà des erreurs fondées sur une connaissance incomplète de l'anthropologie religieuse locale, un choix plus cohérent sur le plan "théo-logique» aurait été possible et une «bonne» correspondance entre le dieu des uns et tel ou tel dieu des autres aurait été pensable. Ainsi, pour les Fang du Gabon, le choix de Mebeghe, disons l'entité cosmique "primordiale ", eût été plus judicieux que celui de Nzame, la figure de l'Ancêtre ${ }^{3}$. Mais la mesure des impasses d'une correspondance cosmo-théologique terme à terme entre les traits substantiels de figures divines, ancestrales ou héroïques décontextualisées, ignore surtout la distance qui sépare les logiques ou les pratiques de la relation aux dieux ou aux ancêtres. Le malentendu avait un caractère structurel et non seulement conjoncturel et le travail syncrétique était amorcé de longue date. 
7 Dans les traditions orales populaires, les propos ironiques et les plaisanteries à propos des contresens missionnaires sur les noms divins sont monnaie courante et largement mis en exergue par les ethnologues. Mais, dans l'ensemble, le sens commun indigène semble s'être beaucoup moins préoccupé de ces détournements du sens originel des noms vernaculaires que les intellectuels savants qui parlent de "manipulation" missionnaire. L'erreur ou les contresens missionnaires n'ont généralement pas empêché que le nom indigène retenu par les catéchismes s'impose à tous comme l'appellation unique et courante de la divinité. Le Nzame des Fang comme le Zamba des Bëti ou le Nzambi du monde congolais ont triomphé des autres appellations plus «authentiques» dont on ne trouve plus de traces paradoxalement aujourd'hui que dans les dictionnaires des missionnaires. La "reprise d'initiative» des peuples colonisés que représentent les syncrétismes et prophétismes indigènes, a dans l'ensemble consacré l'usage missionnaire du nom de Dieu au point de transmuer cette appellation en une pratique inscrite de tout temps dans la mémoire de la tradition africaine. L'enjeu de la dénonciation de l'erreur s'est déplacé, sur ce terrain, vers l'analyse du travail interactif accompli par les catéchismes, travail souvent repris (et même devancé) par les syncrétismes indigènes.

8 Il est plus difficile de qualifier, sans la dénoncer, l'entreprise "savante » qui a véritablement conduit à prendre en otage "nos Négrilles » de la forêt équatoriale pour en faire les témoins « exceptionnels » d'une révélation divine primitive : la «preuve de Dieu par les Pygmées » en quelque sorte. L'apologétique missionnaire est pourtant là encore pleinement en phase avec la tradition indigène locale : les relations de maître à esclave ou de patron à client qui existent de longue date entre les Noirs bantu et les Négrilles n'ont jamais empêché les premiers de combiner mépris et fascination envers les seconds. Sans parler des récits des Pygmées eux-mêmes, qui comme tous les peuples se pensent tout simplement comme "les hommes", tous les mythes d'origine du bwiti ou des cultes du Gabon s'accordent à reconnaître que tout vient «à l'origine » des Pygmées, ces petits hommes de la forêt qui ont transmis aux hommes noirs tous les secrets, et en premier le « secret de la vie et de la mort » révélé par la transmission de l'eboga, la plante sacrée hallucinogène ${ }^{4}$.

9 À l'origine de cette entreprise savante de prise en otage théologique des Pygmées, on trouve essentiellement trois hommes. En premier, la grande figure du «bon et savant » père W. Schmidt, le grand théoricien des «aires de cultures » («Kulturkreis»), le fondateur de la revue Anthropos, qui programmera les missions et expéditions d'enquête ciblant particulièrement les populations pygmées dans plusieurs régions $\mathrm{du}$ monde, de l'Asie à l'Afrique. C'est bien lui qui développe la thèse selon laquelle les Pygmées constituent, malgré leur dispersion géographique et leurs variantes morphologiques, une Urrasse, une race unique pure, tout à fait primitive, les derniers représentants de « l'humanité dans l'enfance ». D'où la priorité qu'il accorde à « l'étude des pygmées... la tâche la plus importante et la plus urgente de l'ethnologie et de l'anthropologie » (Schmidt 1910). Loin d'être assignés au statut de dégénérés, de soushommes, intermédiaires entre les singes et les hommes, les petits hommes se révèlent des génies d'inventivité qui auraient créé d'un seul coup l'arc et la flèche, et bien d'autres choses : la fidélité conjugale du couple monogame, la prière et [...] l'idée de Dieu. Comme le remarque ironiquement M. Mauss (1968: 504): «S'il y a des coups de grâce dans le domaine de la technique, pourquoi n'y en aurait-il pas dans le domaine intellectuel et moral ? »Cette thèse d'une révélation soudaine et primitive de l'idée la 
plus pure de Dieu et des plus hautes idées morales, confirmée par le témoignage des Négrilles, est soutenue dans la série d'articles parus d'avril à décembre 1912 sur "L'origine de l'idée de Dieu» dans la revue Anthropos. Elle sera reprise dans l'ouvrage Der Ursprung der Gottesidee (Schmidt 1912), et poursuivie dans les trois volumes parus en 1926, 1929 et $1931^{5}$.

10 C'est sur le versant religieux qu'intervient en particulier Mgr Alexandre Le Roy (1854-1938), évêque d'Alinda et vicaire apostolique du Gabon, Supérieur de la Congrégation des pères du St Esprit (1896-1926), qui publie en 1905 dans le cadre de la très savante Société de Géographie son ouvrage sur Les Pygmées, Négrilles d'Afrique et Négritos de l'Inde ${ }^{6}$. Son investissement dans la revue Anthropos ${ }^{7}$, ses cours dans le cadre de la chaire d' «Histoire des religions » de l'Institut catholique (décembre 1907-février 1908) ainsi que l'ouvrage issu de ses cours sur La Religion des Primitifs (1909), ont fait de lui un intellectuel catholique engagé, le partisan d'une contre-ethnologie catholique, l'adversaire déclaré d'une science ethnologique universitaire, française et laïque, inféodée aux théories évolutionnistes et matérialistes modernes. Le "Supérieur " Le Roy s'est construit dans les cercles intellectuels parisiens une réputation d'homme de terrain et se prévaut d'une légitimité ethnographique qui s'appuie essentiellement sur ces années passées au Gabon dans les années 1890 (même s'il a aussi une forte expérience de l'Afrique de l'Est depuis 1877 : À travers le Zanguebar [1884]).

11 Mais en Afrique équatoriale, l'homme du terrain, celui qui parle la langue des indigènes, le vrai spécialiste des Négrilles, comme des Fang, c'est manifestement le père Henri Trilles. Comme le rappelle subrepticement ce dernier en soulignant les qualités de "grand écrivain" de son Supérieur, c'est bien lui qui dès 1899 repère des « exemplaires» de Négrilles plus ou moins authentiques et plus tard entre en contact avec des villages pygmées de «l'hinterland du Congo Nord». Trilles conduit Le Roy dans des «campements négrilles » disséminés au cœur de la forêt dans la région du Fernan Vaz ou dans le nord du Gabon : «J'étais, aime à le redire Trilles (1932: 12), son compagnon de voyage et d'étude, et son interprète." La précision sur le rôle d'interprète n'est pas innocente quand on connait le discours de Le Roy sur l'obligation de l'apprentissage des langues pour les « missionnaires de la science ».

12 La grande somme que publie Trilles (1932), vingt ans plus tard, sur Les Pygmées de la forêt équatoriale, est issue d'un cours professé à l'Institut Catholique, alors qu'il est un auteur reconnu comme spécialiste des Fans, notamment avec la publication de l'ouvrage très savant sur Le totémisme chez les Fãn (ibid. : 1912b), préfacé par $\mathrm{M}^{\mathrm{gr}} \mathrm{Leroy}$. La contribution de Trilles prend la place du quatrième volume prévu par W. Schmidt sur les Négrilles d'Afrique et vient en fait répondre à la demande et à la politique scientifique de ce dernier, qui l'a révisé et "épluché », et qui le présente en introduction. Il est publié en même temps que les conclusions d'autres enquêtes programmées par le nouveau directeur (Schmidt), depuis 1926, du Musée missionnaire ethnologique du Latran et financées à partir de 1923 par le Vatican et Pie XI, sur les Pygmoïdes de la Terre de feu, les Négritos des Philippines, les Boschimans, et autres. L'ouvrage de Trilles sort la même année que celui du R. P. Schebesta (1932) sur les Pygmées de l'Ituri, les Aka, les Efé et les Bambuti (Afrique centrale) ${ }^{8}$. On a donc affaire à un vaste chantier ethnographique inscrit dans un "programme scientifique», soutenu par les publications d'une revue et des éditions d'ouvrage, un cycle d'exposition, la fondation d'un Institut Anthropos en 1931, et le prolongement d'un musée, un véritable dispositif (Dietrich 1992). 
13 Le père Schmidt, dans son introduction, présente l'étude de Trilles en soulignant que : «Pour la première fois un groupe déterminé de tribus pygmées [est] étudié par un observateur exercé et consciencieux connaissant leur langue, vivant avec eux et ce pendant de longues années $»^{9}$. Trilles est donc le Malinowski tant attendu des Pygmées. Le résultat est à la fois impressionnant et troublant: Trilles, en dehors des formules convenues d'hommage, prend systématiquement le contre-pied de toutes les conclusions anthropologiques de son "Supérieur» concernant les Pygmées: sur l'organisation clanique et les règles d'alliance, le totémisme et le fétichisme, etc. Il s'évertue surtout à rappeler les conditions douteuses du recueil des données ethnographiques, de leur localisation et de leur interprétation, et à souligner leurs contradictions manifestes. Et en même temps, il ne remet nullement en cause le catéchisme de base sur la primitivité et la pureté du sens moral et religieux de «nos chers Négrilles », et surtout l'argument théologique de la révélation primitive. Il est clair qu'on ne touche pas au dogme de cette ethno-théologie catholique qui a la bénédiction du Vatican et de l'autorité pontificale.

\section{Se situer sur le terrain de l'adversaire : les faits, rien que les faits...}

14 Comme le montre l'analyse des enjeux qui ont entouré la création de la première semaine d'ethnologie religieuse de Louvain en 1912 (Courtois 2004), l'arrière-fond de cette entreprise est celui de la crise moderniste (1902-1907) et l'enjeu est plus précisément celui des réponses à apporter au développement d'une certaine science et histoire des religions. Sur ce plan, Le Roy est sans état d'âme et défend si l'on peut dire une position réaliste et moderne, pas pour autant "moderniste", au regard de la science ethnologique naissante. Il n'est pas question pour lui de céder à l'obscurantisme et à la défiance vis-à-vis d'une histoire des religions qui est en train de se renouveler et de laisser la place vide face au défi de l'ethnologie naissante. C'est l'époque du tournant ethnologique de l'école durkheimienne qui découvre la richesse des matériaux ethnographiques et la possibilité méconnue d'observer la vie primitive sur le «terrain».

Le Roy ne se prive pas dans son livre sur La Religion des Primitifs (1909) de jouer, à la manière de Schmidt, l'ethnologie anglo-saxonne qu'incarne Andrew Lang, auteur de The Making of Religion (1898), contre une histoire des religions à la française, laïque, manifestement soumise aux dogmes du matérialisme évolutionniste. Les représentants de cette dernière vont de Réville, titulaire de la chaire d'histoire des religions au Collège, à Reinach dont le catéchisme totémiste sert de référence au Freud de Totem et Tabou, et qui est manifestement la bête noire de Le Roy. Mauss fera remarquer que l'appareil de preuve des premiers articles de Schmidt sur l'origine de l'idée de Dieu, parus dans Anthropos, est mis au service d'une thèse qui n'est pas nouvelle puisque c'est celle de Lang, déjà fortement discutée dans le monde anglo-saxon à l'épreuve notamment des faits ethnographiques australiens (Mauss 1968: 87) ${ }^{10}$. Les débats scientifiques internes qui existent sur les questions de totémisme ou autres, dans chaque tradition nationale et au sein même du champ anthropologique, sont ici systématiquement ignorés au profit de la focalisation sur tel ou tel auteur plus ou moins diabolisé : il y a ceux qui pensent bien et ceux qui pensent mal. Mauss s'inquiète surtout d'un genre scientifique qui témoigne d'une tendance "manifestement apologétique » et qui n'hésite pas à recourir «à des procédés de discussion que l'on est surpris de rencontrer sous la plume d'un savant, alors même qu'il appartient à une 
congrégation religieuse : par exemple, à la page 524 du tome IV d'Anthropos, on traite deux collaborateurs de l'Année Sociologique d'“auteurs juifs" "... L'antisémitisme de Schmidt faisait partie de son milieu.

Penser bien, c'est surtout faire preuve de «bon sens ». Dans sa discussion des lectures du totémisme comme « la religion primitive de l'humanité », Le Roy (1909:117) - qui sait très bien, lui, ce qu'est le totémisme comme pacte de sang et alliance symbolique puisqu'il a été lui-même « initié » à la chose - en appelle avant tout à la simplicité des faits et des données indigènes que "nos savants compliquent en surajoutant leurs $a$ priori [...] ». L'arme suprême consiste donc à se situer sur «le terrain où nous attire l'adversaire » (ibid.), celui des faits : tous les faits, mais rien que les faits. La nouvelle science ethnologique, partie intégrante de la science chrétienne, doit se construire sur le pari ou le paradoxe d'un positivisme apologétique. Le respect des faits doit pouvoir conduire à admettre que le surnaturel existe comme fondement naturel de la religion, que le miracle d'une révélation primitive peut faire partie des faits rationnellement prouvés de l'histoire, n'en déplaise aux matérialistes et aux évolutionnistes. Les faits premiers qui transcendent la conscience des individus étant les faits de langue, ceux-ci sont au cœur de l'enjeu, d'où la nécessité d'apprendre et d'étudier les langues pour démontrer que la langue est la matrice de l'idée de Dieu, l'expression par excellence de sa présence même éclatée et dispersée dans la pluralité des noms divins.

17 L'adversaire suprême c'est le matérialisme évolutionniste. Comme le dit Le Roy en résumant les thèses de Réville: «Il fallait que l'homme sortant de l'animalité soit d'abord naturiste, puis animiste, puis fétichiste, puis idolâtre, puis théiste » (ibid. : 399). Ces catégories ou concepts, pris isolément, ne sont pas pour l'ethnologue sans valeur descriptive, à condition qu'on ne cède pas à la surinterprétation de ce qui relève de simples "manières de dire ", de légendes et de mythes que les primitifs ne prennent pas eux-mêmes au sérieux et qui n'ont pour eux "aucune signification religieuse " (ibid.: 78). Ce qui est inadmissible pour Le Roy ce sont les théories partisanes qui veulent inscrire ces données dans un schéma évolutionniste de reconstruction de la genèse des religions universelles parfaitement contestable aux yeux de tous les spécialistes y compris « laïcs ». Ce schéma manifestement ne fait pas partie des faits. Mais la posture critique de Le Roy serait plus crédible s'il ne s'empressait pas de soutenir dans la foulée que l'idée de Dieu autant que l'idée d'âme relèvent en revanche des données immédiates de la conscience indigène (ibid.: 143-144), que la conscience religieuse et morale est première, et que c'est elle qui est en fait à l'origine de tout. On se retrouve avec le paradoxe bien connu d'un évolutionnisme à l'envers, d'une histoire de l'humanité primitive marquée par une évolution spirituelle régressive du sens du divin. Le dogme de la révélation primitive est au cœur des enjeux de l'écart entre la pureté et la supériorité des idées religieuses et morales des Pygmées (catholiques sans le savoir), et la dégénérescence qu'incarnent entre autres les Noirs bantu, fétichistes, anthropophages, idolâtres - pour autant pas pires que les Grecs et les Romains, avant la régénération du christianisme.

\section{À chacun son pur Pygmée}

18 La preuve par les Pygmées suppose, et c'est toute la clé de la démonstration, que l'on soit assuré de trouver dans ces "petits hommes» quelques survivants de la pure humanité primitive: "La racine du monde», les «anciens de la terre.» Or sur l'ensemble des fronts de l'enquête, chaque spécialiste soutient avoir trouvé les « vrais » 
Pygmées ${ }^{11}$. Le R. P. Schebesta considère que les Pygmées mbuti de l'Ituri sont les plus authentiques. Le père Trilles le juge bien prétentieux, car ses Négrilles à lui (surtout les Békü) « se donnent le vieux nom égyptien Akka, quatre fois millénaire et qui figure sur les Pyramides ». L'argument de la filiation égyptienne est un lieu commun exploité par Schebesta, même si l'inscription évoquée se trouverait en fait à Beni-Hassan, en Égypte, sur une tombe de la $\mathrm{VI}^{\mathrm{e}}$ dynastie qui représente un nain négroïde ${ }^{12}$. L'Égyptomanie est très présente dans l'œuvre de Trilles puisqu'il laisse entendre, sans contradiction, que la langue, et même la religion, des Fang - qui sont pourtant des Bantu -, sont très proches de celles des Égyptiens, au point qu'on peut sérieusement évoquer «une origine égyptienne du peuple fang " (Trilles 1912a : 247). Les thèses afro-centristes ont été, on le sait, largement préparées par les spéculations missionnaires.

Tous les spécialistes s'accordent aujourd'hui à reconnaître que les populations " pigmoïdes » connues se définissent dans l'interaction avec les populations dont elles dépendent selon des formules très diverses de relation (de maître à esclave, de patron à client, ou d'entente contractuelle) ${ }^{13}$. Surtout le métissage biologique et l'échange linguistique sont la règle (Thomas 1979). Ce constat s'applique particulièrement aux relations entre des Négrilles comme les Békü du Gabon et ce que Leroy et Trilles appellent les Noirs bantu, représentés ici par les Fang. Nos savants n'ignorent pas cette réalité du métissage des Négrilles, au regard des «exemplaires » connus et observés (particulièrement chez les A-jongo du Fernan Vaz auxquels se réfère Le Roy), mais cela ne fait que relancer une quête sans fin de la pureté de la race qui se traduit par des classifications de plus en plus subtiles. Le Roy distingue au moins quatre types de Pigmoïdes : primaire, secondaire, tertiaire, quaternaire, et Trilles situe ses Békü dans le secondaire. Lui-même distingue le type noir, plus grand $(1,50 \mathrm{~m})$, du type jaune cuivré, plus petit $(1,40 \mathrm{~m})$, la petitesse et la couleur claire de la peau étant deux critères de pureté de la race. Ne parlons pas du critère de l'odeur du Négrille qui pour Trilles, faisant écho aux propos des populations voisines, rappelle « l'odeur fade et écœurante de fourmi blanche écrasée » (Trilles 1932 : 39). Le mystère de la création est que Dieu a voulu que la pureté soit associée à la puanteur.

Trilles ne cesse de souligner que le type "negrito pur » de Le Roy renvoie soit à des individus isolés ou des familles vivant dans des villages fang ou autres (comme le fameux danseur Mba-Solé, un informateur privilégié), soit à des campements plus ou moins à l'écart dans la forêt, sachant que dans tous les cas ces Négrilles sont parfaitement «métissés ». L'argument du métissage biologique pourrait naturellement valoir indépendamment du métissage linguistique ou culturel, mais il ne fait pas de doute pour «nos » ethnologues que la pureté de la race va de pair avec la pureté des idées religieuses et morales. Sans renoncer à la quête de "vrais pygmées, non domestiqués, asservis ou métissés, mais vivant en liberté dans leurs forêts, aussi immenses qu'impénétrables " (ibid.: 8), Trilles déplace cependant la frontière en se situant sur le terrain méthodologique, quasi-expérimental, d'une « ethnographie pure, la meilleure ». Celle-ci suppose l'éloignement dans des campements à bonne distance des Noirs bantu et ne peut être que le fait de «celui qui demeure » (Trilles $1945: 17$ ), et pratique le renoncement à tout interprète. Trilles ne se prive pas de pourfendre tous les travaux antérieurs de ceux qui ne font que passer et qui s'appuient sur l'artifice d'une situation où le Pygmée interrogé répond en fait dans la langue de l'interprète (le fang) ou dans la langue d'une tribu où il a vécu. En fait Trilles ne cessera de faire la même expérience puisqu'il parle au départ parfaitement la langue fang à des gens qui 
en général, surtout les informateurs privilégiés (comme le chef de village Ti-Háx), parlent tous cette langue. L'ethnographie pure associée à la maîtrise et à l'usage de la langue « propre » des Pygmées, clé d'accès à la pureté supposée de leur monde, renvoie en fait à des situations d'interlocution très circonscrites et bien spécifiques au sein même de la vie des campements.

\section{Les petits hommes qui prient}

21 Au-delà de la question de la pureté de la race ou de celle de la langue qui est un préalable, il est clair que nos experts des choses religieuses visent avant tout à cerner la pureté de la «vie religieuse» des Pygmées, ces petits hommes "profondément religieux» comme s'empresse de le noter Trilles (1932: 94). Schmidt avait posé d'emblée le principe qui veut que les populations primitives ont de Dieu une idée d'autant plus nette qu'elles sont plus incultes, le dénuement matériel, et même civilisationnel (les plus civilisés n'étant pas ceux qu'on croit), allant de pair avec l'élévation spirituelle.

Dans sa monographie sur les Pygmées, Le Roy parcourt toutes les dimensions de leur vie, mais il est hautement significatif que Trilles insiste, dans sa somme, sur le fait qu'il faut impérativement commencer par la vie religieuse (avant même la langue, ce qui ne manque pas de surprendre). Il republiera d'ailleurs séparément en 1945 la partie exclusivement consacrée à la vie religieuse dans un ouvrage au titre évocateur : L'âme du Pygmée d'Afrique. L'enjeu est de taille puisqu'il ne s'agit pas seulement de sauver des âmes innocentes mais de marquer d'emblée l'écart absolu des Pygmées par rapport aux « singes » : les singes ne prient pas Dieu. Et plus surprenant, les Noirs bantu non plus. Toute la démonstration religieuse repose alors sur ce glissement qui est censé avoir conduit de la pureté des Négrilles à la dégénérescence des Bantu: anthropophages, féticheurs, idolâtres, des prédateurs qui ne prennent même pas la peine de remercier Dieu de ses bienfaits par quelque prière ou sacrifice, comme lors de la récolte du miel en forêt. Le paradoxe est que ce sont ces sauvages qui traitent traditionnellement les Négrilles comme des « singes $»^{14}$.

Le Roy avait d'emblée esquissé dans La Religion des Primitifs la trame de cet argumentaire qui n'a rien de très original. Le monde surnaturel des Bantu, contrairement à ce que certains ethnologues ont pu soutenir, n'exclut pas l'idée de Dieu. Ils ont comme tous les primitifs la conscience "nette » et "immédiate ", l'intuition claire de quelque chose comme un Être suprême, une Cause première ou même un "Dieu créateur ", mais cette conscience "nette » s'accompagne d'une idée « incorrecte » ou en tout cas moins élaborée que celle que les théologiens (catholiques) ont mis en forme. Les diverses et multiples appellations de cette entité (Celui qui conçoit, juge, pense, fabrique toute chose) peuvent être lues comme les «attributs divins" d'un même Dieu qui n'a pas à vrai dire de nom propre. L'idée que ces appellations plurielles puissent renvoyer à des catégories d'action ou des énonciations situées, sans comporter la moindre signification substantielle, n'est évidemment pas à l'ordre du jour. Toute tentative pour aller plus loin sur ce sujet (où habite ce dieu ? qu'y avait-il avant lui ? peut-il mourir ?) se heurte de toute façon, comme le reconnaît Le Roy (1909 : 184), à l'indifférence : « Mais au fond c'est là pour les indigènes une question oiseuse, insoluble et sur laquelle chacun peut penser ce qu'il veut. » Le champ est donc libre pour penser à leur place. 

est indifférent aux hommes, et qu'il ne peut en aucun cas être un interlocuteur fiable et surtout être sollicité par quelque culte que ce soit. Le Roy aime citer pour illustrer cette attitude un extrait de conte recueilli par Trilles en milieu fang: "Dieu c'est Dieu, l'Homme c'est l'Homme. Chacun sa maison. Chacun chez soi. » Ce compartimentage se retrouvera aussi dans la réponse à l'offre missionnaire : Manière de Blancs, Manières de Noirs. La conséquence de cette posture «théologique » est que pour tout ce qui touche à leurs affaires et leurs intérêts quotidiens, les Noirs bantu misent sur le culte des fétiches, des ancêtres ou des génies, plus ou moins impersonnels, et qui ont leur force propre. Tout ce que "les ethnologues" ont rangé sous les termes de naturisme, fétichisme, animisme, polythéisme ou même totémisme, trouve ici une pertinence incontestable. Mais le processus qui conduit à fétichiser des objets, à personnifier des êtres naturels (soleil, lune), à déifier des êtres humains, est la conséquence d'une perte de sens du divin et de l'idée pure de Dieu, et relève d'élaborations secondaires et dérivées. C'est bien là la thèse de l'évolutionnisme à l'envers qui participe malgré tout, soit dit en passant, du « fétichisme de l'évolution » avec lequel Le Roy prétend pourtant en finir, au nom de la fidélité aux faits (ibid. : 349).

Les Négrilles, comme on pouvait s'y attendre, auraient au contraire conservé « une connaissance sensiblement plus claire que celle d'autres races supérieures » de l'idée pure d'un dieu « personnel et souverain » (Le Roy 1928: 350 ; Trilles $1932: 88)^{15}$. Ce dieu ne peut être confondu avec la manière dont sa puissance se manifeste dans ses créations (le soleil, la lune, le tonnerre) et il n'est point question ici d'adorer des astres ou de rendre un culte à des idoles. Le fétichisme (les amulettes et autres), la sorcellerie et l'anthropophagie, même aux yeux des Négrilles, ce sont les mœurs des Noirs bantu, et une condamnation aussi «nette » des pratiques dégénérées des autres ne peut que plaider en faveur de l'élévation de leurs sentiments moraux. Le point essentiel et décisif est que les Négrilles ont «la connaissance ou le pressentiment d'une vie future impliquant récompense ou châtiment » (Le Roy 1928), donc l'idée miraculeuse d'un dieu moral et rénumérateur qui s'intéresse à la vie et à la conduite des hommes. En retour, les petits hommes de la forêt n'hésitent pas à rendre grâce à Dieu, naturellement, par un culte très simple, par la prière spontanée, l'offrande des premiers fruits de la nature ou le «sacrifice » d'une part de la chasse, en veillant à ce que «la part de Dieu » lui revienne, sans demande de contrepartie, en dehors de tout esprit intéressé ou d'attente de contre-don... un « sacrifice d'amour », selon l'expression de Trilles (1932: 89).

\section{Une ethnographie de « bonne foi »}

Le plus étonnant c'est que les convictions avancées sur la «théologie des Négrilles » par Le Roy s'appuient sur des témoignages extrêmement ténus, pour ne pas dire inexistants. Surtout que l'auteur lui-même s'évertue à brouiller les pistes. Ainsi le dieu des Békü a le même nom que le Nzame de leurs maitres fang ; le Nzambi des A-jongo du Fernan Vaz est clairement selon leurs dires l'Anyambié de leurs voisins nkomi. Le témoignage individuel sur la récompense des bons et le châtiment des méchants après la mort (les bonnes âmes s'enfouissant sous terre, et les méchants étant jetés par Dieu dans le feu d'en Haut [sic]), est extorqué chez les A-jongo - des «populations de négrilles métissés qui prétendent avoir conservé leurs traditions primitives » (Le Roy 1928 : 179-180) -, sans la moindre critique (comme s'en étonnera Mauss lui-même). Dans le même temps, le témoignage d'un Mba-Solé, du groupe des Békü, sur la 
condamnation des méchants dans l'Ototolane, est clairement interprété comme soumis à l'influence des Fang (totolane c'est en effet l'enfer ou le purgatoire des catéchismes fang [ibid.: 184]). Trilles (1932: 71), revenant lui-même sur des sources et des informateurs qu'il connaît bien, enfonce le clou en déclarant à propos de ce "pygmée de race pure vivant chez les Fang": "Les traditions et les récits qu'il donne ne ressortent en aucune manière du fond négrille, elles sont nettement et uniquement bantu.»

Sur le terrain du culte où se déplace l'enjeu, ce qui représente pour Leroy: «Le témoignage de la haute religiosité des Négrilles, témoin millénaire du sacrifice offert par l'homme aux premiers âges du monde, témoignage touchant des humbles enfants de la terre, au Père qui est aux cieux ", correspond en réalité à des rites très classiques d'offrandes pratiqués lors de la récolte du miel ou des noix de nkula en forêt (le noyer indigène), une "fête des prémices" comportant comme partout l'obligation de réserver en premier leur part aux dieux ou aux ancêtres. Le comble en la matière est que Trilles - qui déclare ne jamais avoir assisté lui-même à cette cérémonie de cueillette de nkula en forêt (même s'il s'en ait fait confirmé tous les détails) - signale que le récit de cette grande fête saisonnière, repris et "si bien raconté par $\mathrm{M}^{\mathrm{gr}}$ Leroy " comme une "procession " (sic) entamant une "sorte de cantique ", est en fait un récit de seconde main transmis au père Trilles par l'abbé A. Raponda, le premier prêtre gabonais d'origine mpongwé (Trilles 1932 : 98). Le refrain de ce cantique pygmée n'a d'ailleurs rien de négrille puisqu'il est justement en langue mpongwé. Quant aux traductions en français des chants qui accompagnent les départs pour la cueillette ou la chasse, elles reprennent sans retenue le lyrisme des cantiques des processions catholiques : «En avant, en avant pour cueillir le présent du Seigneur ${ }^{16}$.

Le procédé relève clairement de l'habillage chrétien de rites indigènes qui est une pratique courante chez nos ethnographes catholiques. Trilles (1912a: 246) lui-même n'hésitera pas, sans le moindre scrupule, à publier dans Chez les Fang ou Quinze années de séjour au Congo français comme illustration du culte du byeri des Fang, une photo empruntée à la collection du R. P. Augouard représentant un étal de crânes et de boîtes «à byeri » relevant clairement d'un style caractéristique des statuettes des traditions sango. Cette indifférence manifeste aux écarts distinctifs des styles ethniques va de pair avec un déni de la valeur esthétique de ces statues qui enchantent aujourd'hui nos collectionneurs ( « une grossière et hideuse statue, taillée à grands coups de hache dans un informe tronc de bois noir [...]»). Mais s'ajoute surtout un pur et simple habillage (par une couche surajoutée de peinture blanche), du féticheur sango (photographié dans la collection Augouard dans sa nudité $)^{17}$, en officiant vêtu d'une sorte de surplis blanc familier de la liturgie catholique. Ce «blanchiment» de la nudité noire commande le programme de la description autant qu'il inspire la mission d'évangélisation. 
240. Officiant du culte des ancêtres. Sango, docum. R.P. Augouard (fin XIX siècle)

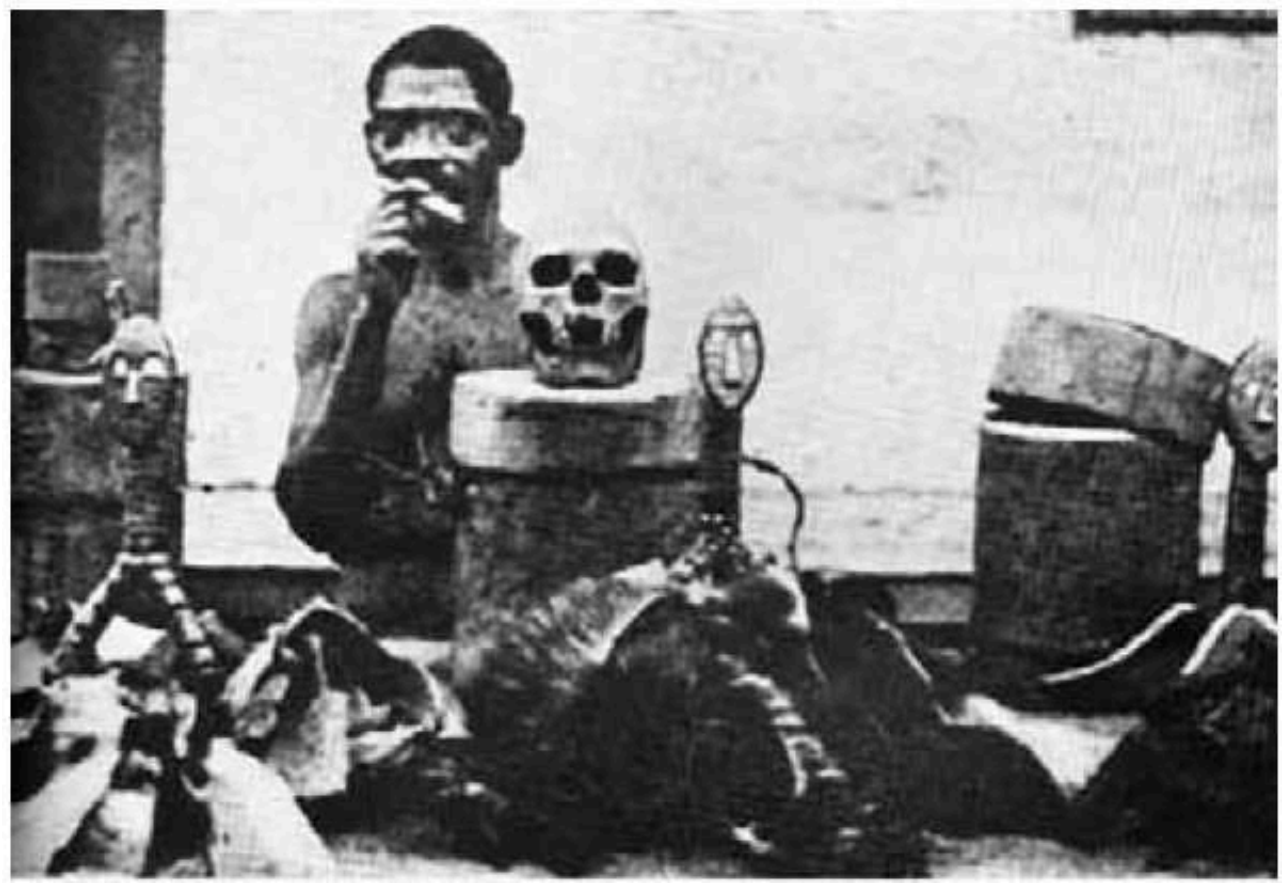

Source : Perrois (1979: n²40).

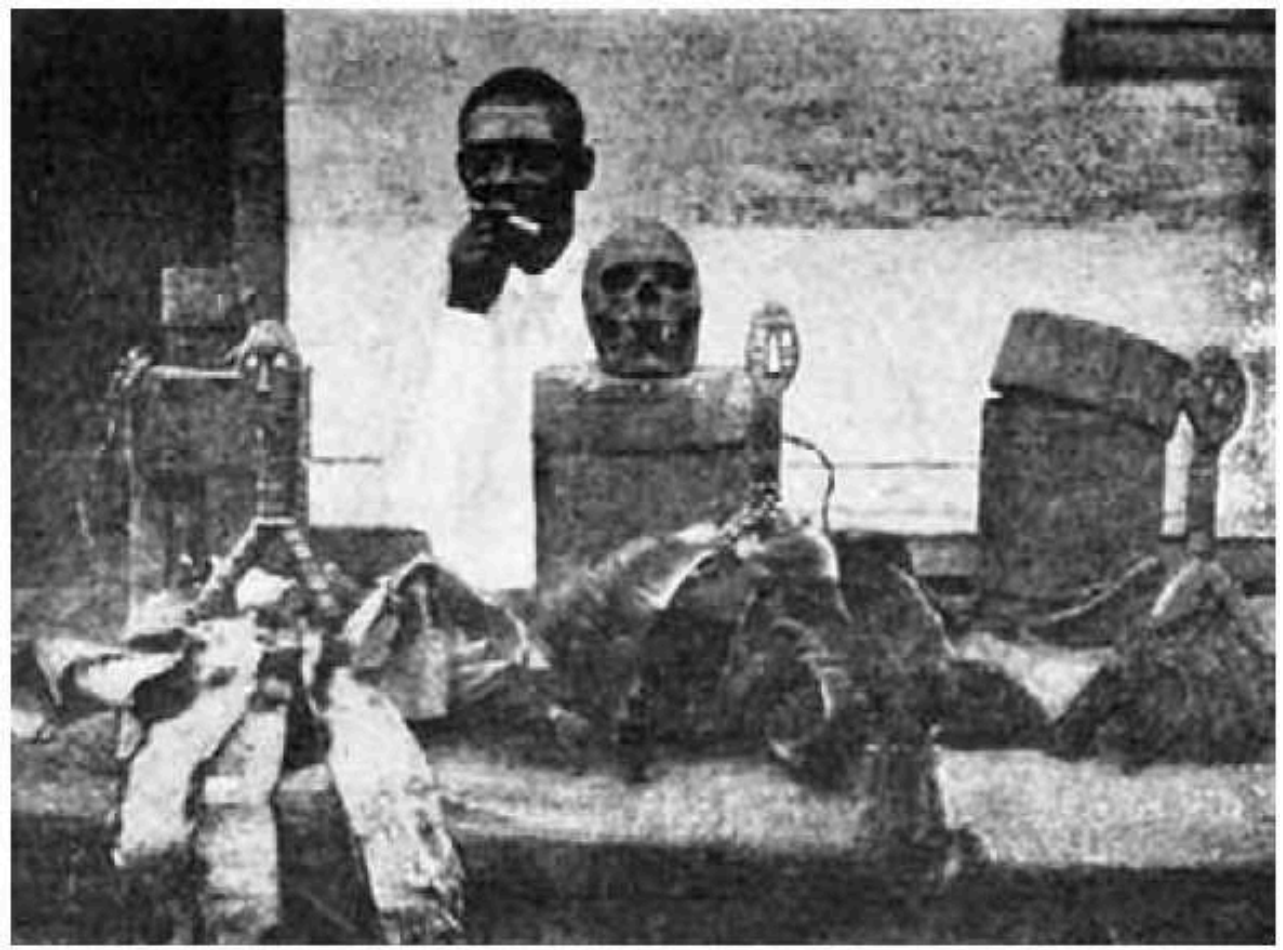

Source: Trilles (1912a: 246)

Trilles entremêle, comme à son habitude, dans le commentaire de la "procession » des Pygmées au pied de l'arbre nkula, des spéculations sur l'origine égyptienne du nom donné au noyer $(\mathrm{ku})$, visant à confirmer le destin à part de ce peuple des Négrilles perdu au milieu des Bantu, à un sens réel du détail ethnographique. Ainsi, il s'interroge dans des termes dignes de l'analyse structurale, sur le fait que, lors de ce rite, la première 
noix que l'homme grimpant à l'arbre prend entre ses dents soit "avortée » et que le grimpeur redescende la tête en bas: fruit anormal, conduite anormale? ou offrande d'un fruit mauvais aux esprits d'en bas?

\section{Le fond négrille et la « compénétration des races »}

'anthropologie sociale et religieuse du monde pygmée revisitée par Trilles accentue le trouble. Dans l'ensemble, la somme considérable des informations dont semble disposer Trilles, ses compétences linguistiques, et surtout le sens critique qu'il développe l'amène à prendre sur tous les plans le contre-pied des thèses de Le Roy: sur l'organisation clanique et le totémisme, sur l'ignorance du fétichisme et de la sorcellerie, sur les cultes et les "ministres du culte», sur le monde des esprits et la mythologie, sur le nom de Dieu, etc. La conclusion qui s'imposerait à la lecture est surtout celle d'une profonde continuité métissée entre le monde pygmée et le monde bantu.

31 Et cependant, Trilles (1932: 64), même vingt à trente ans plus tard, à distance du terrain, reprend le catéchisme de base de cette entreprise, même si la formulation en devient franchement problématique : «En somme, les Négrilles se distinguent de leurs voisins par des croyances, qui, assez semblables en première apparence, sont en réalité beaucoup plus simples, beaucoup plus épurées, ou mieux encore, très catégoriquement beaucoup plus primitives ${ }^{18}$. Sur la langue, un enjeu majeur, où Trilles est bien placé pour trancher, et où la similitude du lexique est évidente, cela ne l'empêche pas d'évoquer un "vieux fond négrille ", une "trame très ancienne ", un filet dont les mailles sont usées, et les pièces rapportées (ibid. : 218). Le plus significatif peut-être est le terrain des contes, légendes et proverbes, d'une grande richesse, et la spécialité de Trilles, son apport ethnographique majeur ${ }^{19}$. La conclusion se fait ici plus explicitement (plus catégoriquement) incertaine : «De par sa contexture, ses mots archaïques, tel chant, conte et légende semble bien appartenir au vieux folklore négrille ", mais... "Démêler l'origine de façon certaine, affirmer que tel proverbe ou chant est certainement pygmée, tel autre douteux, tel enfin importé, est absolument impossible» (ibid. : 264, n. 1). La " compénétration des races", pour reprendre ses termes, semble bel et bien un fait irrémédiable.

Sur le plan de la complexité de la vie religieuse, Trilles apporte des données partielles qui pourraient rejoindre les thèses de Turnbull $(1965$ : 252) sur la vanité de la recherche d'un système cosmogonique cohérent et unifié. Mais la question de savoir si ce peuple "profondément religieux» a conservé le «flambeau des révélations primitives transmises par le Créateur " (ibid. : 502) est désormais clairement reconnue comme une question théologique qui ne peut trouver de réponse au plan ethnographique. Le relevé infini des noms de dieux ou la description des rites des «prémices» ne peut faire que l'ethnographie puisse combler l'abîme des origines. Le mérite de Trilles est de donner à entendre, souvent entre les lignes, les impasses de cette entreprise ethno-théologique dont il reproduit le credo. La première difficulté, bien connue, tient au fait que les Négrilles, encore plus que les Bantu, ne se posent pas les questions ultimes que se posent les Blancs, et manifestent même une franche irritation face à leur insistance. Aux interrogations de l'ethnologue sur la manière dont Dieu récompense le bien ou répond au problème du mal, les réponses sont claires: "Tu nous fatigues vraiment ! Demande nous comment on prend un poisson dans l'eau, une bête dans la forêt, là le 
chemin est tout droit (facile). Mais tout ça! Tiens regarde nos femmes, tu vois, elles préparent le ngon [un plat préparé avec la pulpe farineuse des pépins d'une espèce de citrouille] : pan, pan, pan! Elles frappent sur les pépins de citrouilles. Tout ce qu'il y a dedans sort, mais regarde les frapper ! pan, pan! pauvre citrouille ! Toi, tu es la femme et nous la citrouille. » Et quand on passe à la question de savoir si Dieu peut mourir, là : « Tout le monde se met à rire [...] et c'est la seule réponse » (ibid. : 75).

Lorsque l'ami informateur privilégié joue le jeu du " philosophe » ou du «théologien » négrille comme Mba-sole, ce dernier bricole manifestement dans l'interlocution une réponse qui puise dans la pluralité amalgamée des ressources locales et s'inspire par anticipation, et en toute lucidité, des «manières de Blanc ». Car la qualité du conteur, nous dit-on, consiste à imaginer et à broder à partir d'une trame pour se faire valoir auprès de l'auditoire. Bel exemple, là encore relaté en toute honnêteté, par notre ethnographe missionnaire, des récits d'origine des races fabriquées à partir de terres de couleurs variées, noire, jaune, et... pourquoi pas blanche. Les Blancs, les revenants, ne sont-ils pas depuis longtemps présents dans l'imaginaire des Négrilles? Ces derniers ne cessent d'ailleurs de se demander pourquoi les Blancs posent de telles questions, eux qui savent déjà tout.

À défaut de faire parler les Négrilles, on peut interroger cette langue "propre " qui parle pour eux puisqu'elle conserve, selon Trilles, la mémoire de leurs liens très anciens avec les Égyptiens. Mais sur ce terrain, comme sur les autres, les questions ultimes se perdent dans les méandres et les impasses de la traduction des paroles ancestrales qui alimentent les prières, les chants, les invocations ou incantations. Le Roy notait déjà que les « prières " primitives sont généralement des " paroles cabalistiques, des mots et des phrases obscures, des allusions difficiles à saisir par notre esprit européen, des archaïsmes enfin qui viennent des âges lointains, dont la signification est même perdue, mais que l'on garde fidèlement, et qui sont jugés d'autant plus efficaces qu'on les comprend moins» (Le Roy 1909 : 297). Trilles (1932 : 97) confirme cette conclusion : "Les mots plus ou moins déformés ne sont pas du langage courant, et restent fort difficiles à comprendre, même pour les négrilles. » Selon lui, la répétition de ces incantations ne s'accompagne d'aucun culte du mystère des origines, le rythme des danses en l'honneur du soleil ou de la lune l'emporte manifestement sur le sens énigmatique des paroles. Le mystère qui se montre dans le rituel n'est rien qui puisse se dire.

\section{De l'ethno-fiction au faux ethnographique}

Ce chantier ethnographique équatorial d'une science catholique au service de la preuve de l'universalité de la vérité du catholicisme (prenant en otage des Pygmées «naturellement» catholiques) apparait véritablement surréaliste. Mais une telle entreprise d'investissement imaginaire du Pygmée comme paradigme d'une humanité pure, porteuse de l'idée originelle (et universelle) de Dieu, véhicule tous les paradoxes d'une époque donnée. En ce tout début du $\mathrm{xx}^{\mathrm{e}}$ siècle, l'obsession d'une race pure aux confins des origines de l'humanité va de pair avec la réalité de la «compénétration des races» et l'évidence réitérée d'un continuum de populations métissées. L'histoire témoigne que ce sont les sociétés et les époques travaillées par le métissage qui multiplient les classements et les gradations du pur et de l'impur, en se donnant les 
indicateurs d'une mesure de l'origine. Pour nos ethnologues missionnaires, la taille, la couleur et l'odeur des Pygmées ne trompent pas. Mais comment savoir si l'origine «égyptienne » des Fang est plus authentique que celle des Pygmées? Ces intellectuels catholiques militants assimilent leur combat contre les dérives païennes de la modernité occidentale et de la France laïque qui « tue Jésus dans l'âme de ses enfants » (Trilles 1912a: 257) à la sauvegarde de cette humanité dans l'enfance protégée miraculeusement de la dégénérescence qui affecte leurs voisins bantu passés sous l'emprise du fétichisme et de l'Esprit du Mal. La preuve de l'existence au cœur de l'Afrique des Ténèbres d'une humanité témoignant d'une innocence antérieure devait fonder l'espérance d'une régénération morale et spirituelle des "pauvres âmes » de l'Afrique centrale, selon l'expression de Monseigneur Augouard (Coulon 2001 : 100).

La conversion de nos experts des choses religieuses à l'épreuve du «terrain », leur foi soudaine dans le culte positiviste des faits purs, sont associés au recueil de données de "première main », la voie par excellence de la révélation primitive. Dans le cas des populations pygmées, cette ethnographie pure se confond avec la recherche de campements isolés au cœur de la forêt, et donc de lieux non contaminés par la civilisation. Le mythe occidental des "premiers contacts " avec l'humanité primitive rebondit, au plein cœur d'une situation coloniale qui confirme, aux yeux des missionnaires, le degré de corruption dont l'humanité occidentale est capable et donne tout son sens à l'enjeu de la rédemption de l'âme des Pygmées.

Cette entreprise d'ethnologie " catholique " peut facilement, avec le recul, tomber sous le coup de la critique des ethno-théologies qui procèdent à la surinterprétation théologique des données ethnographiques. Le bilan scientifique de ce chantier reste à faire ${ }^{20}$, même si la plupart de ses acteurs ont fini par reconnaître, au moins entre les lignes comme Trilles, que les questions théologiques appellent des réponses théologiques, et que Dieu n'est pas « un fait religieux », surtout pas pour les Pygmées. Mais dans sa réponse aux critiques de Radcliffe-Brown concernant la crédibilité ethnographique de ses informateurs missionnaires et le biais chrétien de ses thèses sur les croyances des populations des îles Andaman, Schmidt persiste et signe : il met en cause non seulement la présence sur le terrain et la compétence linguistique des informateurs de Radcliffe-Brown, mais surtout le parti pris métaphysique de l'anthropologie évolutionniste des ethnologues britanniques associé à une posture d'incroyance qui ne permet pas d'accepter que des primitifs insulaires puissent avoir une haute idée de Dieu (cité par Brandewie 1983 : 111-116). Evans-Pritchard, une de nos plus grandes autorités ethnographiques, loin d'être indifférent à la thèse du monothéisme primitif, lui fait écho en suggérant que sa découverte chez les Nuer d'une forme de religiosité supérieure à celle de ses concitoyens n'est pas sans rapport avec l'expérience religieuse de sa conversion au catholicisme ${ }^{21}$. Le "tournant linguistique " et le relativisme post-moderne nous ont familiarisé depuis avec la déconstruction du positivisme ethnographique et nous connaissons aujourd'hui d'autres prises d'otages d'aimables sauvages au service de nos idéaux occidentaux. On pense aux artefacts de l'entreprise ethnographique qui a pu conduire la jeune assistante de Boas, Margaret Mead, à offrir le miroir de la sexualité des adolescentes de Samoa aux fantasmes de la société américaine (Tcherkézoff 2001). La production de "faux ethnographiques» au service de la « bonne cause » n'est pas le privilège des missionnaires.

L'exemplarité de l'invention ethnographique du Pygmée catholique comporte néanmoins un tel engagement théologique, et repose sur une telle pétition de principe, 
qu'on ne peut la confondre avec l'ensemble des contributions de l'ethnographie missionnaire. Amateurs ou professionnels, de nombreux travailleurs de la preuve en terre de missions ont pratiqué le dédoublement, la coupure ou le refoulement par rapport à leurs convictions éthiques et théologiques, et refusé la confusion des langues. Mais le discours professionnel de la dénonciation et de la manipulation des données tend à laisser place aujourd'hui à son envers, l'éloge du missionnaire "ethnologue accompli $»^{22}$. Le parti pris de la réhabilitation de la «science des pères » trouve un allié objectif dans le relativisme épistémologique et la conversion au perspectivisme ${ }^{23}$. L'anthropologie apologétique de nos ethnologues missionnaires vaudrait bien, après tout, l'anthropologie romantique et participante d'un ethnologue comme C. Turnbull. L'autorité ethnographique de l'anthropologue professionnel, et surtout son monopole, n'étant plus de mise, il devient possible de soutenir que les intérêts de profession qui ont chacune leur "mission » doivent être traités sur le même plan, en un mot qu'un texte en vaut un autre. On finirait par gommer l'écart entre une description interprétative de situations observées ou le recueil de témoignages en contexte d'énonciation, et le recours à des données de seconde ou de troisième main formatées pour les besoins de la démonstration. On mesure surtout le malentendu qu'il y a dans cette affaire à ignorer le " point de vue indigène », en l'occurrence celui de l'ethnologue catholique, qui ne doutait pas, pour sa part, de l'universalité absolue de la vérité qu'il détenait. Comme le disait Bourdieu, le relativiste oublie simplement que l'indigène n'est pas relativiste. On peut parler néanmoins d'une nouvelle forme du « paradoxe $d u$ missionnaire » : si l'entreprise « scientifique » n'a pas atteint son objectif sur le terrain où elle pensait gagner, la bataille théologique de la vérité, elle, a contribué malgré elle à la production interculturelle (ou inter-textuelle) du pur Pygmée (catholique ou écologique) comme figure de l'autre scène de l'imaginaire moderne des populations africaines.

\section{BIBLIOGRAPHIE}

AUGÉ, M., 1982 Génie du paganisme, Paris, Gallimard.

Bahuchet, S., 1993 « L'invention des Pygmées », Cahiers d'Études africaines, XXXIII (1), 129 :

153-181.

BAHUCHET, S. \& THOMAS, J.-M.-C., 1981 « L'organisation religieuse », in S. BAHUCHET, J.-M.-C. THOMAS \& A. EPELBoIn (dir.), Encyclopédie des Pygmées aka, Paris, Éditions du CNRS ; Paris-Leuven, Peeters : 169-214.

Bourdieu, P., 1987 « Sociologie de la croyance et croyances des sociologues », in P. BOURDIEU, Choses dites, Paris, Éditions de Minuit : 106-111.

Brandewie, E., 1983 Wilhem Schmidt and the Origin of the Idea of God, Lanham-New York-Londres, University Press of America.

Coulon, P., 2001 « Le catholicisme et la vapeur du centre de l'Afrique », Mémoire Spiritaine, 14, deuxième semestre : 68-111. 
Courtois, L., 2004 « La première semaine d'ethnologie religieuse à Louvain en 1912. Les débuts difficiles d'une démarche progressiste sur fond de crise moderniste ", in O. SERVAIS \& G. VAN'T SPIJKER (dir.), Anthropologie et missiologie. XIX ${ }^{e}-X^{e}$ siècles. Entre connivence et réalité, Paris, Karthala : 95-118.

Dietrich, S., 1992 « Mission, Local Culture and the "Catholic Ethnology" of Pater Schmidt », JASO, $23(2): 111-125$.

Dupré, W., 1975 Religion in Primitives Cultures, A Study in Ethnophilosophy, La Haye-Paris, Mouton.

Evans-Pritchard, E. E., 1956 Nuer Religion, Oxford, Clarendon Press.

-, 1972 [1932] Sorcellerie, oracles et magie chez les Azande, Paris, Gallimard.

Laburthe-Tolra, P., 1985 Initiations et Sociétés secrètes au Cameroun, Essai sur la religion beti, Paris, Karthala.

-, 2000 « L'ethnologue Alexandre Le Roy (1854-1938) », Mémoire Spiritaine, 12 : 56-81.

LAGAE, O. P. (M $\left.{ }^{\mathrm{gr}}\right), 1926$ Les Azandé ou Niam-Niam, Bruxelles, Vromourt et Co.

LANG, A., 1898 The Making of Religion, London, Longmans-Green and Co.

LE ROY, A. $\left(\mathrm{M}^{\mathrm{gr}}\right), 1884$ À travers le Zanguebar, Lyon, Bureau des Missions Catholiques ; Paris, Congrégation du Saint-Esprit.

-, 1909 La Religion des Primitifs, Paris, Beauchesne.

—, 1928 [1905] Les Pygmées, Négrilles d'Afrique et Négritos de l'Inde, Paris, Beauchesne.

Lévi-Strauss, C., 1962 Tristes Tropiques, Paris, Plon.

MARY, A., 1999 Le défi du syncrétisme. Le travail symbolique des Prophètes d'Eboga, Paris, Éditions de l'EHESS.

,- 2000 «Conversion et conversation : les paradoxes de l'entreprise missionnaire ", Cahiers d'Études africaines, XL (4), $160: 779-799$.

Mauss, M., 1968 Euvres I, Paris, Éditions de Minuit.

мвот, J. E., 1975 ebughi bifia, « Démonter les expressions ». Énonciation et situations sociales chez les Fang du Gabon, Paris, Institut d'ethnologie-Museum national d'histoire naturelle, Mémoires XIII.

Museur, M., 1969 « Récentes perspectives sur la culture des Mbuti », Cahiers d'Études africaines, IX, $33: 150-159$.

PEEL, J. D. W., 1995 « For Who Hath Despised the Day of Small Things ? Missionary Narratives and Historical Anthropology », Comparative Studies in Society and History, 37 (3) : 581-607.

Perrois, L., 1979 Arts du Gabon, Paris, ORSTOM (« Arts d'Afrique Noire »).

Philippart De Foy, G., 1984 Les Pygmées d'Afrique Centrale, Marseille, Éditons Parenthèses.

Piskaty, K., 1957 « Ist das Pygmaën Werk von Henri Trilles eine Zuverlässige Quelle ? », Anthropos, $52(1-2): 33-48$.

ROSA, F., 2003 L'âge d'or du totémisme. Histoire d'un débat anthropologique (1887-1929), Paris, CNRS Éditions-Éditions de la Maison des Sciences de l'Homme. 
Sahlins, M., 1979 « L'apothéose du capitaine Cook », in M. IZARD \& P. SMITH (dir.), La Fonction Symbolique, Paris, Gallimard : 307-343.

Sawada, M., 2001 « Rethinking Methods and Concepts of Anthropological Studies on African Pygmies' World View : The Creator-God and the Dead ", African Study Monographs, suppl. 27 : 29-42.

Schebesta, P. J., 1925 « Die Forschungsexpedition von P. Paul Schebesta SVD in 1924/ 25 bei den Semang-Pygmäen und den Senoi-Pygmoiden auf der Halbinsel Malakka », Anthropos, 20 : 718-739.

-, 1932 Bambuti, die Zwerge vom Kongo, Leipzig, Brockhaus.

-, 1940 Les Pygmées, Paris, Gallimard.

-, 1952 Les Pygmées du Congo Belge, Bruxelles Inst. Roy. Col. Belge.

SCHMIDT, P. W., 1910 [1905] Die Stellung der Pygmœen Vœelker in der Entwicklungsgeschicte des Menschen, Stuttgart, Strecker und Schröder., 1912 Der Ursprung der Gottesidee, Münster, Aschendorff.

SINGLETON, M., 1972 « Theology, "Zande Theology” and Secular Theology in Zande Themes », Essays presented to Sir Edward Evans-Pritchard, Oxford, Blackwell : 130-157., 2001 " Expansionnisme ecclésiastique et expertise ethnologique : le Cardinal Lavigérie et les Pères Blancs en Tanzanie », Neue Zeitschrift für Missionswissenchaft, $1: 15-32$.

TCHERKÉZOFF, S., 2001 Le mythe occidental de la sexualité polynésienne 1928-1999 : Margaret Mead, Derek Freeman et Samoa, Paris, PUF.

THIEL, J. R., 1977 Ahnen, Geister, Höchste Wesen : Religionsethnologische Untersuchungen in Zaïre-KasaïGebiet, St Augustin bei Bonn, Anthropos.

THOMAS, J.-M.-C., 1979 « Emprunt ou parenté. À propos des parlers des populations forestières de Centre Afrique ", in S. BAHUCHET (dir.), Pygmées de Centrafrique. Études ethnologiques, historiques et linguistiques sur les Pygmées «Ba.Mbenga » (aka/baka) du nord-ouest du Bassin congolais, Paris, SELAF («Études Pygmées, III ») : 141-169.

TRILLES, H., 1912a Chez les Fang ou Quinze années de séjour au Congo français, Lille, Desclée, De Brouwer et Cie.

-, 1912b Le totémisme chez les Fãn, Munster, Anthropos.

-, 1932 Les Pygmées de la forêt équatoriale, Paris, Bloud \& Gay.

-, 1945 L'âme du pygmée d'Afrique, Paris, Éditions du Cerf.

-, 2002 [1905] Contes et légendes fang du Gabon, Paris, Karthala.

TURNBULL, C. M., 1960 « The Molimo : A Men's Religion Association among the Ituri Bambuti », Zaïre, XIV (4) : 307-340.

-, 1965 Wayward Servants : The Two Worlds of the African Pygmies, New York, American Museum of Natural History.

VANDENBERGHE, A., 2006 « Entre mission et science. La recherche ethnologique du père Wilhem Schmidt : SVD et le Vatican (1900-1939) », Le Fait Missionnaire, Missions et Sciences Sociales, 19, décembre : 15-36. 


\section{NOTES}

1. Je me permets de renvoyer ici pour quelques éléments de ce débat à MARY (2000 : 779-799), à l'article de PEEL (1995) ainsi qu'à SINGLETON (2001). Un grand merci à Mike Singleton pour ses remarques et ses informations de première main sur les enjeux d'un milieu qu'il a bien connu. Ce texte doit beaucoup aux échanges du colloque organisé à Louvain-la-Neuve en 2005 autour de son parcours biographique et de son œuvre.

2. Pour une discussion classique sur le sujet illustrée par la transformation du dieu Mawu, principe femelle du panthéon dahoméen, en dieu masculin, vieux et barbu, voir AUGÉ (1982 : 133-135).

3. Tout en reconnaissant le non-sens d'un retour en arrière, P. LABURTHE-TOLRA (1985: 28) note que : «Les termes de Ntondobë (ou Mebe'e) expriment immédiatement avec moins d'équivoque (que celui de Zamba) la pureté et la profondeur du concept de Dieu chez les anciens Bëti »(mes italiques). Où l'on voit que le débat sur le vrai nom de Dieu engage tout simplement le débat sur la question du monothéisme originel.

4. Sur le rôle des Pygmées dans les mythes d'origine de cette plante sacrée au sein de la tradition du bwiti, voir MARY (1999).

5. Pour une étude d'ensemble des présupposés scientifiques de l'œuvre et des activités de Schmidt, voir BRANDEWIE (1983).

6. L'ouvrage de $\mathrm{M}^{\mathrm{gr}} \mathrm{A}$. LE ROY, Les Pygmées, Négrilles d'Afrique et Négritos de l'Inde (édité par Mane en 1905, réédité par Beauchesne en 1928), est d'abord paru sous forme d'articles dans les Missions catholiques de Lyon, dès 1897.

7. Il écrit le liminaire du premier numéro de la revue à la demande du père Schmidt : «Le rôle scientifique des missionnaires », 1906.

8. Un rapport sur l'expédition de Schebesta chez les Twa de Malakka (Philippines) a déjà été publié dans Anthropos en 1925 (sCHEBESTA 1925).

9. Préface à TRILLES (1932: XIII). Faut-il préciser qu'il s'agit là d'un effet d'annonce ? Les conditions évoquées par Trilles d'une ethnographie participante de longue durée dans les campements pygmées n'ont jamais été réunies dans le cadre de sa présence au Gabon de 1883 à 1907.

10. Sur ces débats, voir ROSA (2003).

11. Sur le thème de «l'invention des Pygmées ", voir entre autres la mise au point de BAHUCHET (1993).

12. Voir PHILIPAART DE FOY $(1984: 17)$ qui se réfère au numéro de la Revue historique de 1891. BAHUCHET (1993 : 167) conteste la présence du nom Akka sur cette inscription tombale représentant un « nain » qui danse divinement, il s'agirait plutôt d'un Donka.

13. Voir entre autres l'inversion des lectures en termes de domination de TURNBULL (1965).

14. Voir le sens très discuté de l'appellation békü par laquelle les Fang les désignent (TRILLES 1932 : 146).

15. Tous les anthropologues qui ont travaillé depuis chez les Pygmées akka ou mbuti (TURNBULL 1965 ; BAHUCHET \& THOMAS 1981 ; SAWADA 2001) considèrent que ce culte rendu à un dieu unique et bienveillant est une pure invention: la vie "spirituelle » y est essentiellement dominée par la référence au monde de la forêt, lieu d'une puissance 
dont dépend toute vie et toute mort, dont tout vient et où tout retourne, les chants et les rites s'adressant uniquement aux esprits des morts qui habitent ce lieu.

16. On est bien loin des chants de la cérémonie molimo de "réveil de la forêt » des Mbuti décrite par tURNBULL (1960 : 191-216) et également relatée par SCHEBESTA (1952: 267-277).

17. L'original de la photo avant son «blanchiment» est reproduit dans PERROIS (1979: $\mathrm{n}^{\circ} 240$ ).

18. Mes italiques.

19. Sur Trilles comme traducteur-producteur des contes fang, voir la préface de J. E. Mbot à la réédition de Contes et légendes fang du Gabon (TRILLES 2002).

20. On trouve des éléments de bilan critique de l'ethnologie religieuse missionnaire pygmée, surtout à vrai dire sur le chantier de longue durée de Schebesta, citons entre autres : THIEL (1977), BAHUCHET (1993), SAWADA (2001). Sur Trilles en particulier, voir PISKATY (1957) qui jette le doute sur ses écrits, au cœur même des publications d'Anthropos. Pour toutes les raisons évoquées dans cet article, la faisabilité d'une confrontation ethnographique comme celle qui peut être faite à propos des descriptions et interprétations de Schebesta (MUSEUR 1969), est littéralement impensable dans le cas de l'ethnographie de Trilles, et pas seulement parce qu'elle précède de trente ans celle de Schebesta sur d'autres terrains (BAHUCHET 1993). Seules les ethno-philosophies des Pygmées continuent à puiser dans de telles sources, voir DUPRÉ (1975).

21. Dans sa préface à Nuer Religion, l'anthropologue fait ce premier aveu : "Those who give assent to the religious beliefs of their own people feel and think, and therefore also write, differently about the beliefs of other peoples from thoses who do not give assent to them » (EVANS-PRITCHARD 1956: VII), mes italiques. M. SINGLETON (1972) qui a fait sa thèse avec E. P. sur le «monothéisme nilote » confirme l'influence directe du « théisme nuer » sur la re-conversion de l'anthropologue.

22. Sur Le Roy comme « ethnologue accompli », voir P. LABURTHE-TOLRA (2000).

23. Voir les conclusions de Michel museur (1969: 157) qui prêche, dans sa lecture de Schebesta et de Turnbull, pour une « objectivité » résolument relativiste pratiquant la juxtaposition des perspectives, sans confrontation empirique, comme si l'addition des points de vue était par elle-même une source de progrès de la connaissance.

\section{RÉSUMÉS}

Résumé

Cette contribution porte sur la production à la fois savante et militante d'une ethnologie catholique au service d'une anthropologie apologétique prenant le contre-pied de la culture universitaire et scientifique de son époque. Les travaux de $\mathrm{M}^{\mathrm{gr}}$ Le Roy et du R. P. Trilles sur la religion « primitive » des Pygmées d'Afrique équatoriale sont une des applications du programme initié par Wilhelm Schmidt, le fondateur de la revue Anthropos et le père du monothéisme 
primitif. Cette ethnologie religieuse engagée par des missionnaires, connaissant le terrain, visait à faire des campements de «nos petits Négrilles» une sorte de "terrain apologétique ». Elle participe de la surinterprétation ethno-théologique des données ethnographiques mais elle est minée dès le départ par la contradiction d'une démarche qui vise à apporter la preuve sur le terrain ethnographique d'un dogme théologique. Elle s'est déconstruite d'elle-même en provoquant le grand rire des Pygmées pris en otage par Dieu et ses pères. La réhabilitation ambiguë des écrits de la Science des pères sur fond de relativisme oblige à poser le problème du recours au faux ethnographique dans cette entreprise apologétique.

\section{Abstract}

This paper deals with Catholic ethnology research, both knowledgeable and militant, at the service of apologetic anthropology that took the opposing view to that held by the academics and scientists at the time. The works of $\mathrm{M}^{\mathrm{gr}}$ Le Roy and R. P. Trilles on the "primitive" religion of the Pygmies in equatorial African is one application of the programme launched by Wilhelm Schmidt, father of primitive monotheism and the founder of the review Anthropos. The religious ethnology carried out by missionaries who knew the field, aimed to turn camps of "our little picaninnys" into a kind of "apologetic terrain". It contributed to the ethno-theological overinterpretation of ethnographic data in that period, but was undermined from the start by the contradiction that lay in an approach aiming to provide proof of a theological dogma in ethnology. It deconstructed itself by provoking the amusement of the Pygmies themselves, hostage as they were to God and his priests. The ambiguous rehabilitation of the works of the Science des Pères against a relativist backdrop, requires us to raise the issue of using false ethnography in this apologetic enterprise.

\section{INDEX}

Mots-clés : Pygmées, Trilles, ethno-théologie, missionnaire catholique, monothéisme primitif Keywords: Pygmies, Trilles, Ethno-Theology, Catholic Missionary, Primitive Monotheism

\section{AUTEUR}

\section{ANDRÉ MARY}

Centre d'Études Interdisciplinaires des Faits religieux, EHESS, CNRS, Paris. 